\title{
Discover DaVinci: Blockchain, Art and New Ways of Digital Learning
}

\author{
Marko Suvajdzic ${ }^{*}$, , Dragana Stojanovic ${ }^{2}$ \\ ${ }^{1}$ Digital Worlds Institute, University of Florida, Gainesville, FL, 32601, USA \\ ${ }^{2}$ Faculty of Media and Communication, Singidunum University, Belgrade, 101801, Serbia
}

\begin{tabular}{l} 
A R T I C L E I N F O \\
\hline Article history: \\
Received: 16 July, 2021 \\
Accepted: 20 September, 2021 \\
Online: 14 October, 2021 \\
\hline Keywords: \\
Blockchain \\
Art \\
Gamification \\
\end{tabular}

\begin{abstract}
A B S T R A C T Discover DaVinci is a novel augmented reality system that incorporates blockchain technology with experiential learning to engage participants in an interactive discovery of Leonardo da Vinci's oeuvre. The software was created by Marko Suvajdzic, first author of this paper, and it was produced at the University of Florida Digital Worlds Institute. In the true spirit of this "Renaissance man", Discover DaVinci explores new ideas and technologies "ahead of their time", opening up questions about usage of blockchain system in the domain of art and technology. This paper discusses some of these questions, such as relation of art and technology, usefulness of blockchain system for digital art, and new materiality of art in digital and informational age. Proposed work of this manuscript is to present the field of digital learning through a general review and more specifically through a prism of Discover DaVinci project created by Digital worlds Insititute at the University of Florida.
\end{abstract}

\section{Introduction: Art and Technology, the Unseparable Pair}

Speaking about art in contemporary times, together with a still present idea about its alleged autonomy [1], we may tend to understand it as framed within a particular institutional, academic or actual scene [2]. If we concentrate on its manifestations on the stage, in galleries or museums, where we experience it as a certain kind of a screen given to the audience, it is easy to completely miss its deep, continual and historical relation to technology and experimentation. In the other words, as traditional art audience, we often regard art as an object to be watched, listened to, assessed, valued or sold/bought. In such a constellation, the art audience is left in a quite passive state, although art, as a communicational model of encoding and decoding (note the obvious relation to technology and programming techniques!), offers its audience much more [3].

One of the dimensions of art production and placement that is explored in the beginning of 21st century is certainly connected to contemporary communicational, digital and web technologies. It may seem as a new field to be questioned and explored, but the relation of art and technology goes a lot more back into history. It is enough to recall a philosophical term technē, which was used to refer to a making or a doing, but it was also used to designate a kind of an episteme, and it was a name for knowledge in the widest

\footnotetext{
${ }^{*}$ Corresponding Author: Marko Suvajdzic, Email: marko@digitalworlds.ufl.edu
}

sense [4], [5]. What is more important here is that it was used for any kind of artistic practice that involved making-doing, as well as gaining knowledge through technological experimentation. It was particularly used for crafts and mechanical art, but Larry Shiner argues that it might not have been strictly about art as such, but much more about human ability to make and perform, or, more directly, to use technology to improve the possibilities of makingdoing, of understanding art, and of presenting it to others in a concrete historical context [6].

It seems that the current context in which matters of art and art production are just being developed and innovated belongs to digital reality, or, even more forward, to a technological and artificial intelligence (AI) environment. In these digital circumstances, the focus is transferred from an art-object to an artinformation, and from human to machine agency, but it does not mean excluding human factor from the art worlds, institutions and economies - on the contrary. We may say that by introducing machine intelligence and transactional systems, their human users are getting chance to consider these changes as motivational and developmental factor not only in the field of technology, but also in the fields of social sciences and art.

One of the technological platforms, or, better to say, a technological system that is found right at the crosspoint of mathematics, sociology, economy, law and politics is blockchain 
system. As this paper will propose and as we will show in further discussion using Discover DaVinci project as an example, the usefulness of blockchain system for the world of art is, although contested, quite prominent and more and more explored - not only within new forms of art production, presentation, protection, marketing and trading, but also within new forms of learning about art in contemporary times. Researches in this field might prove important not only for technology-oriented studies, but also for the education and learning studies.

\section{Blockchain and Art: Is It All About the Money?}

Looking back to beginnings of blockchain system application, it is obvious that it was created as an "electronic payment system based on cryptographic proof instead of trust..." [7]. This means that blockchain was first and foremost meant to be a more secured and complex system for monetary transactions. This system is based on cryptographically carved currencies of which the first one was Bitcoin, after which it was followed by many more. Blockchain system is thus a distributed database whose security, paradoxically enough, lies in an increase of parties included. So, instead of a trust in one mediator as a representative of a desired transaction, there are hundreds and thousands of nodes, mutually connected, that carry the information about the transaction. The security factor is ensured by peer-to-peer system (P2P), where the server is shared between all the computers and included parties not only the parties involved directly in transaction, but also all the parties that embody this complex system of humans, codes and computational nodes. What is interesting here is that by increasing the number of involved participants we decrease the risk of a problematic transaction. This is a very specific cooperation of machines and humans, working together on the task of transferring information and ensuring that it is not copied or doubled illegally. The information is condensed into a block, and blocks are packed into chains (thus the name blockchain). This is done through a hash function, which is a form of cryptography that functions as a suture between blocks, and it is placed in such a way that it guarantees that the information within a block cannot be changed internally or externally without the complete change being detectable in all the chain nodes. The only, though theoretical way of manipulating blockchain system lies in an option that at least $51 \%$ of elements in the chain decide to manipulate the chain in the same way, and in an exact same time. From here we conclude that the more peers in the blockchain network, the less possibility of manipulating it in any kind of way. On the other side, some downsides of blockchain system include work efficiency problems and high resource consumption due to the process of mining, and that is currently in the focus of research of all those who work on blockchain system inclusion in any of the fields it may find its usage [8].

But is the blockchain system really all about money, and why is its decentralized mechanics so important for the world of art, and, more so, for the process of learning about art? First of all, blockchain system is a system - which means, a specifically operating platform of communication. In this way, any value we put in it, money, art or otherwise, gets included into a specific, mechanical, human-machine built world, a network-like system in itself. Although some can argue that blockchain system is well outside of traditional institutions, and those arguments are given in a very strong way [9], it seems that blockchain system in itself forms a specific set of communicational and transactional rules that inscribes itself in the digital entities it works with. In the sense of previous understanding of art institutions and art worlds [10], it definitely skips the logic of material art exhibition and valuation, but it creates a new world for art existence and presentation, or, more precisely, it adds a new, digital, immaterial layer to previously known logics of art creation, trading and consumption. Thus we cannot really speak about the new, potentially foreign system to the world of art, but of a new technological option for art development and market.

Speaking of that, blockchain system is not only about the art market, although it is a big part of innovation it brings. Of all the other benefits it may bring, besides better possibilities to protect digital artworks and authors' rights, blockchain system offers many options for solving the issues of copying digital works [11]. It also helps promoting yet unestablished authors, and it can resolve the issues of returning material artworks to the countries where they originated from, or to the individuals that have rights to its ownership - in the same time not disturbing work of museums and galleries that currently exhibit these works. Of course, and on the top of it all, blockchain system offers new possibilities of learning about art, especially through gamification strategies. But, before we delve deeper into a concrete example of this possibility that will be described through the analysis of Discover DaVinci project of the University of Florida Digital Worlds Institute, there is still enough space to think through another perspective that the blockchain system brings to the world of art, and that is the issue of (a new) materiality of art in the domain of digital art.

\section{Digital Age and New Materiality of Art}

In her text "Collections Management on the Blockchain: A return to the Principles of the Museum" Helen Kaplinsky mentions posthuman museums in the context of human-machine, or human-algorithm dialogical or network interaction [12]. Mentioning posthuman museums (and maybe we can extend this into proposing a thought of posthuman classrooms?), she poses the question which can be condensed into the following: do we still need bodies in the age of digital and blockchain platforms prevalence? And, how it will influence the world of art? Or, more broadly said, do we still need materiality of the artworld? As Martin Zeilinger says, "Digital art can thus help us rethink questions related both to the materiality of the digital and to the immateriality of the art object as an abstract container of value" [13]. But how is digital art linked to a material, and an art object to immaterial in this quotation? It seems that a digital contemporaneity, and, especially blockchain technology is contributing further to the complexity of this issue, becoming a relevant and interesting topic not only for art itself, but also for the theoretical discourses around art.

Looking briefly into the nature of digital art, we may easily conclude that digital art is a perfect example of an ephemeral, immaterial art practice - so much that it is actually hard to pinpoint the storing "place", or even a "source" of a digital artwork - be it because of its many copies and shares, or due to its (non)physical, digital qualities. It is important to note that digital art is not the first attempt to immaterialize the artwork and 
art practices itself - that attempt can be traced back to conceptual art, as an institutional critique towards capitalist ideologies, as a way of disintegration of traditional art norms, and also as an experimentational practice and exploration of art in a dematerialized, "post-aesthetic" condition [14]. It was in 1973 when Lucy Lippard hoped that “'conceptual art' would be able to avoid the general commercialization... of modernism” [15]. Since then, it seems, we can trace a specific hope in the act of dematerializing art, in the means that in that way it is thought to be able to escape commodification logic of the market, and avoid becoming a high-efficiency container for commercial value". In the age of digital art, the first example of this sort is certainly net art, and for a couple of decades digital art, as a continual line drawn from net art subverting practices, was regarded as an art that is able to contradict the logic of institutionalized and monetized art world. Furthermore, it was regarded as "temporary autonomous zone with no tolerance for institutional dogma and the ideologically suspect economic value systems propagated by institutional bureaucracies of the art world". Perhaps that is why there is still so much resistance towards blockchain technology within the scope of digital art practices, as well as among digital artists and digital art theoreticians. These resistance voices usually point to the dangers of hypercommodification of art; also, they are worried about the possibility of creating a technologically dependent society, or of strengthening the capitalist logic in the domain of art [16]-[20].

To be able to understand relations between digital art and blockchain technology, we have to go back to possible definitions of digital art. According to Christiane Paul, digital art is "art that is digital-born, computable art that is created, stored and distributed via digital technologies and uses the features of these technologies as a medium" [21]. Similarly enough, Hui defines digital objects as appearing to human users "as colourful and visible beings. At the level of programming they are text files; further down the operating system they are binary codes; finally, at the level of circuit boards they are nothing but signals generated by the values of voltage and the operation of logic gates" [22]. Both the authors stress the informational element of the digital object, or digital art object that needs digital technology to be able to exist, or to present itself to the audience. This certainly opens up the path for thinking about contemporary digital technologies, together with blockchain technologies, as relevant factors of enhancing the digital media. In the end, in order to serve and inspire digital art and artists, blockchain system should be both medium and subject of artistic practices, both conventional and critical/subversive. In order for this to happen, artists should have a voice in how this technology might be applied to the field they act in, but to be able to gain this voice, they would have to say yes to technology, and to research the ways it could work for their ideas, and not against them. All the different artworks of emerging blockchain artists already show this, and they successfully overcome duality between human and machine factor, as well as between materiality and immateriality of the artwork (see Figure 1 as an example, also [23]).

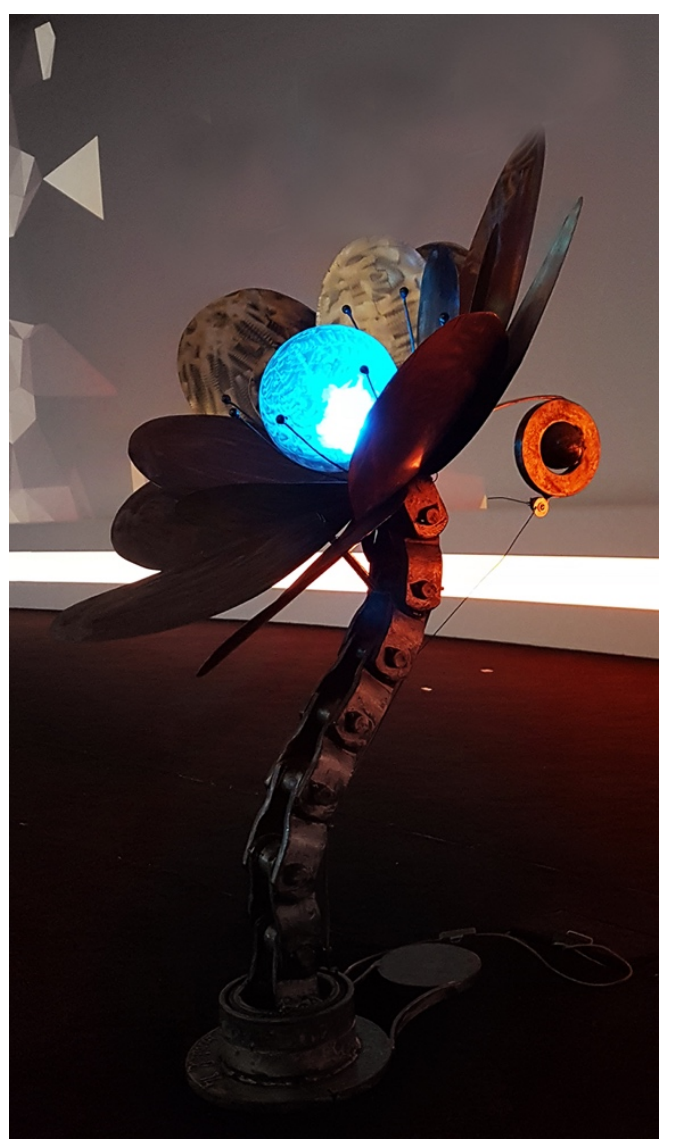

Figure 1: Primavera De Filippi, Plantoid

As innovations in art are always linked to innovations in technology and vice versa, and as making of art, in its quality of techne is always in the same time a knowledge creation, the authors of this text will propose a model of technology research in art, or, differently said, of art research in technology. One of the ways to do it is to work on the intersections of technology, art and learning, and to create gamification strategies of learning about art through blockchain system. One of such examples is Discover DaVinci project, an interactive system produced at the University of Florida Digital Worlds Institute.

\section{Introduction to "Discover DaVinci” Project}

In order to illustrate the emerging potential at the intersection of art and blockchain, we present a case study of a new interactive system produced at the University of Florida Digital Worlds Institute. This software was created by Marko Suvajdžić, associate professor and associate director of Digital Worlds Institute. Suvajdžić's experience includes various digital startups and educational projects involving artificial Intelligence-intensive video game titles for major corporate clients. Suvajdžić also cofounded 5 of his own startups.

The technologies of mobile computing, augmented reality (AR), and blockchain are starting to merge, creating new opportunities and scenarios to interact with our environment. In AR we can look at virtual objects superimposed within a real environment and resize them, rotate them, explore and interact with them on multiple levels. With the combination of AR and blockchain, we can create a system capable of keeping track of 275 
digital assets located virtually in 3D space (i.e., spatial computing). The global scale of blockchain and related technologies heightens the potential for trade and digital distribution with a fully automated and trusted way to keep track of their creations without a "middle-man".

Discover DaVinci is a novel educational tool that teaches concepts of blockchain technology through an augmented reality experiential learning game.

\section{Discover DaVinci Project Partnerships}

This project was developed in collaboration with several units from the University of Florida and industry partners:

- Digital Arts \& Sciences Faculty (Computer Science and Digital Worlds Institute)

- Digital Worlds Studios' Artists and Programmers

- Gator Blockchain Club (gatorblockchainclub.com) - Studentrun blockchain club at the University of Florida

- Center for Innovation and Entrepreneurship (College of Business)

- Creative Campus Committee at the University of Florida

Industry Partners:

- DLUX, decentralized content network (dlux.io)

- Steem (steem.com), and Steemit (steemit.com)

- A-Frame, web VR platform (aframe.io).

\section{Research Study: "Discover DaVinci”}

Discover DaVinci (Fig.2) utilizes the format of a digital, collectible trading \& drafting card game with AR elements on the STEEM blockchain. Although each player "owns" their cards, all transactions are public. This is enabled by the blockchain system itself, that defines private and public key for every user. The private key always stays private and cannot be recovered, and the public key is visible to the others. In this way blockchain system guarantees all the participants both their privacy, and in the same time public visibility of every change in the chain, being enacted by purchases and transfers in ownership.

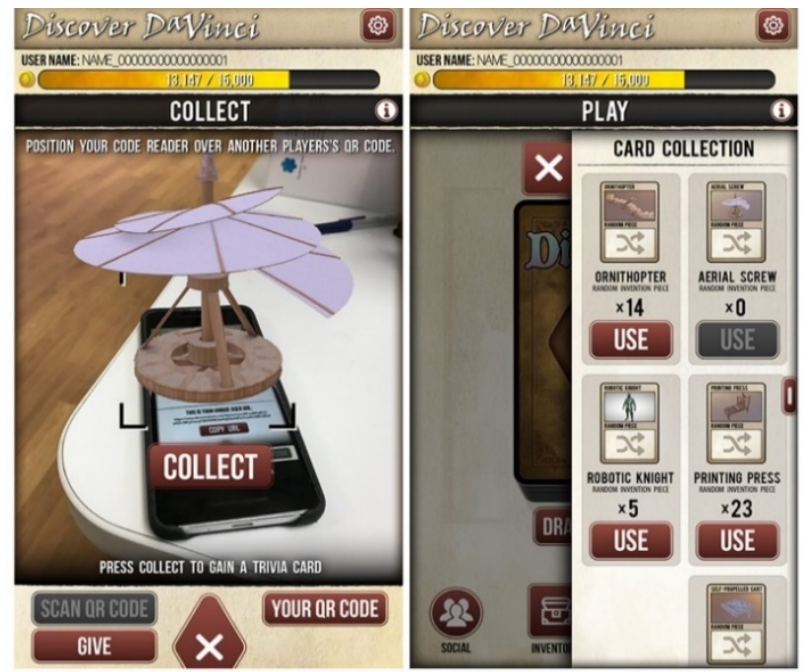

Figure 2: Discover DaVinci game interface, University of Florida (2019)
Discover DaVinci collectible cards are all unique tokens, owned by the player - a digital asset registered to the player's account. The aim is to draw new question cards daily, answer the questions about Leonardo DaVinci, collect the special AR invention cards, and ultimately submit the accumulated card collection into a drawing for prizes. The app was developed to honor the 500th anniversary of Leonardo DaVinci by promoting new and innovative technologies. In the same time, this project is an homage to Leonardo DaVinci's fascination with art and technology, which is an oeuvre of the artist of the Renaissance period, but it may also be a quality of artists maneuvering their own artwork pathways in the age of blockchain technology development.

By using a novel combination blockchain, and spatial computing/AR technologies, the Discover DaVinci mobile app allows users to explore holographic images of DaVinci's devices. By combining AR with blockchain technology, we have created a system capable of assigning unique values and user comments to these digital assets, thus providing a personal sense of ownership, the potential for trade, and a fully automated and trusted way to keep track of these digital artifacts (e.g. collected 3D objects in AR space). Finding the artifacts themselves is an act of discovery, further enhanced by subsequent investigation, collection, and sharing of the artifacts across a diverse social media ecosystem.

The sense of ownership, and not only accumulating of goods is provided by the technology of non-fungible tokens (NFTs), which is especially valuable in the field of art. In the same time, using NFT in tokenization of an artwork or a collectible makes it possible to distinguish the art token from any other cryptovalue, or a cryptocurrency, which are defined as fungible tokens. In a way, this gives us an answer to a previously posed question - is blockchain based art only about the money? Following the logic of non-fungible tokens, it is not. NFTs are not interchangeable, nor convertible in any other value. They possess their own unique value - just as it is the case with material, traditional art. In this way we can see that blockchain system can be regarded as the next step of efficiency and inspiration material within the already existing art forms, art market and art institutions. Of course, as there is always a room for critique and resistance in traditional art world, it is the same in technological world. That is why it is important for the artists and art/art theory teachers to delve deeper into blockchain technology research, and to tailor new media to their own expressions and needs - just as it has always been, as it is shown in the case of Leonardo DaVinci.

Back to Discover DaVinci project, the blockchain system was implemented using the STEEM API, which handled the writing of new user accounts and the reward transactions to the Blockchain (Fig. 3 bottom). The front end was developed as a web app that rendered the various $2 \mathrm{D}$ and 3D/AR components of the game (Fig. 3 top right). Finally, the authentication and account creation was handled using a third-party service, called STEEM Ninja (Fig. 3 top left). 


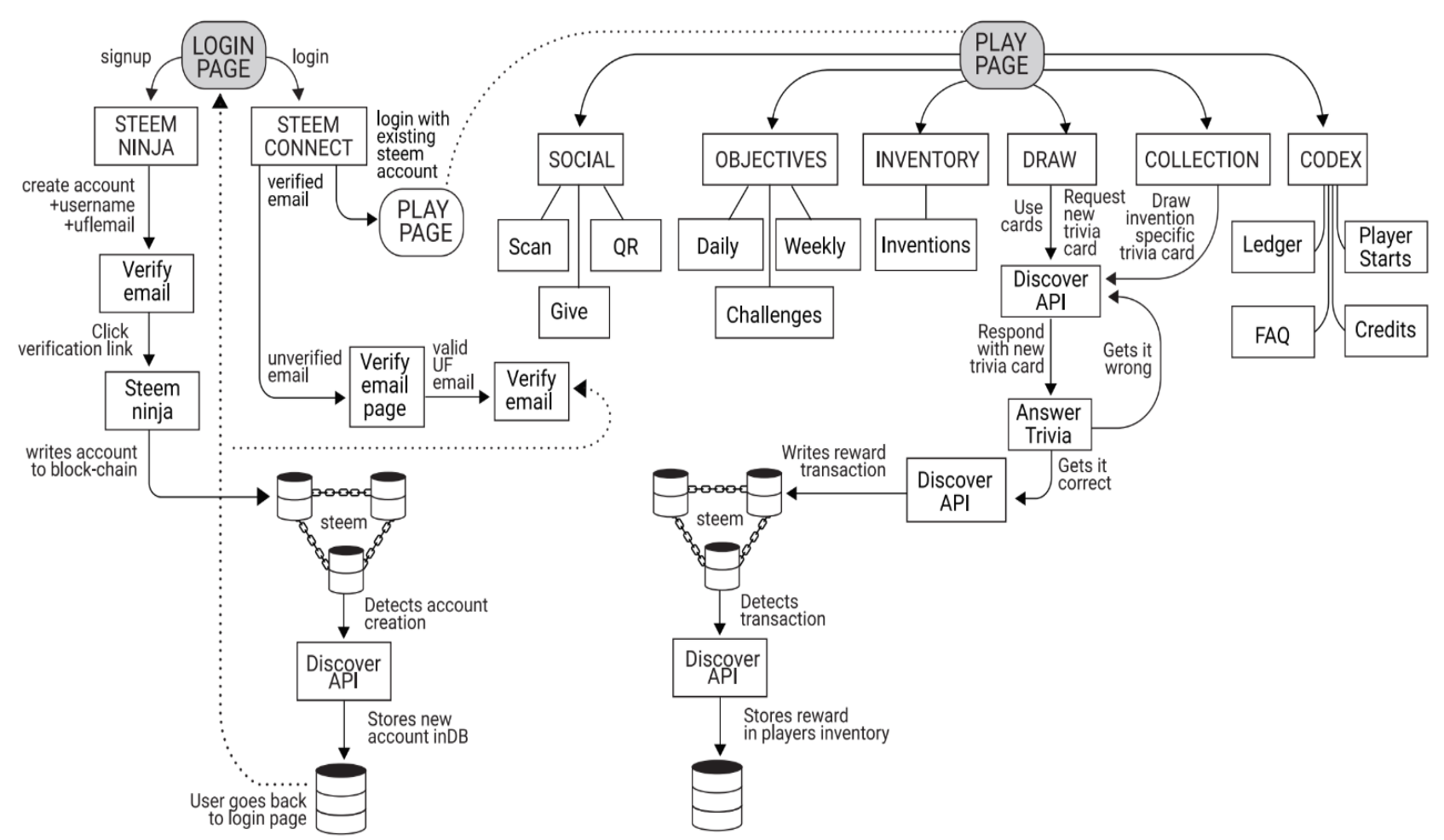

Figure 3: Discover DaVinci-System Architecture illustration, University of Florida (2019)

\section{Discussion}

We launched Discover DaVinci app to the initial audience of University of Florida students and faculty in November of 2019. Over the period of 60 days, we engaged $~ 150$ participants who played the game, and left their feedback on the project's discussion board.

The player feedback has been analyzed, and the subsequent versions in the "Discover" series are currently being evaluated. The project is intended to continue as a platform for gamified education that promotes participant interaction, and supports focused learning on a topic of choice.

In this review paper with the main example of Discover DaVinci App, the project conducted by the team at the University of Florida, Digital Worlds Institute, the authors wanted to refer to Discover DaVinci application and its potential within the fields of gamification and learning concepts. Certainly, there is still a space for further tests to be done in the future, so we could effectively reveal the impact of such an application on learning methods and contemporary educational approaches involving blockchain technology. However, at this point, the authors would like to propose a formal conclusion, stating that these kinds of applications offer effective possibilities for understanding teaching and learning processes within generation that adopt knowledge at the present moment. What has been noticed is certainly an ease of a new technology adoption in the entertaining educational setting, and that is the point from which we can continue to think about the intersection of technology and education.
Discover DaVinci is an innovative app that uses blockchain technology as a tool that utilizes gamification of education, specifically focused on presenting life and works of Leonardo DaVinci. The app has been custom created for this purpose by the team at the Digital Worlds Institute at the University of Florida, and as such is not directly comparable with general educational tools on the market. However, it is used to illustrate how blockchain technology can be introduced to new users through gamified content.

We believe that this novel confluence of art, technology, and digital communications represents a tangible example of how artists and cultural researchers can leverage the potential of diverse digital resources to support both creative development and cultural inquiry. The Discover DaVinci app allows a diverse player community to utilize their existing mobile devices and participate in acknowledging DaVinci's life work while exploring a new paradigm of digital convergence. The outcomes included an enhanced cross-campus appreciation of personal creativity, social and cultural engagement, and a working framework that exemplifies how emerging technologies can serve esthetic ends while also ensuring fair use and exchange of intellectual property in the digital age.

\section{Acknowledgements}

The authors would like to thank the students and staff who contributed to the development of the app: Liam Wood, Paul Burgermeister, Steven Ettinger, Mark Giles, Jan Zamojski, Kelvin Chang, Ryan Trimble, Nick Heitzman, Seung Hyuk Jang, Anna 
Walters, Kristen Eldridge, Anthony Monteagudo, Aaron Karlson, Chelsea Cantrell, Gabriel Coleman, Jared Crane, Justin Marlin, Tim Difato, and members of the UF Bachelor of Arts in Digital Arts and Sciences Society. We would also like to express our appreciation to the University of Florida Creative Campus Committee for providing funding for this project through the catalyst fund program.

\section{References}

[1] R. Cronk, "The Pseudo-Autonomy of Art," Art on the Rebound: A Collection of Essays on Art and Culture, https://www.westland.net/venice/art/cronk/autonomy.htm, 1996, Retrieved 23 March 2021.

[2] G. Dickie, Aesthetics, An Introduction, Pegasus, 1971.

[3] R. Rojcewicz, The Gods and Technology: A Reading of Heidegger, State University of New York Press, 2006.

[4] D. Carpi, Bioethics and Biolaw through Literature, Walter de Gruyter, 2011.

[5] L. Shiner, The Invention of Art, University of Chicago Press, 2001.

[6] S. Nakamoto, "Bitcoin: A Peer-to-Peer Electronic Cash System," www.bitcoin.org, Retrieved 22 August 2020.

[7] N. Chalaemwongwan, W. Kurutach, "State of the Art and Challenges Facing Consensus Protocols and Blockchain," ICOIN 2018, 957-962, 2018. doi: 10.1109/ICOIN.2018.8343266.

[8] S. Ren, "The Application of Blockchain and its Advantage to the Field of Art," 2nd International Symposium on Big Data and Applied Statistics, Journal of Physics: Conference Series, IOP Publishing, 2019.

[9] R. Myers, "Blockchain Poetics," Catlow R., Garret M., Jones N, Skinner S. (Eds.), Artists Re:Thinking the Blockchain, Torque Editions \& Furtherfield, 239-250, 2017.

[10] A. Danto, "Symposium: The work of Art, the Artworld," The Journal of Philosophy 61(19), 571-584, 1964. doi: 10.2307/2022937.

[11] R. O'Dwyer, "Limited Edition: Producing artificial scarcity for digital at on the blockchain and its implications for the cultural industries," Convergence: The International Journal of Research into New Media Technologies, Sage, 1-21, 2018, doi: 10.1177/1354856518795097.

[12] H. Kaplinsky, "Collections Management on the Blockchain: A return to the Principles of the Museum," Catlow R., Garret M., Jones N, Skinner S. (Eds.), Artists Re:Thinking the Blockchain. Torque Editions \& Furtherfield, 261274, 2017.

[13] M. Zeilinger, "Everything you've always wanted to know about the Blockchain*(*but you were afraid to ask Mel Ramsden)," Catlow R., Garret M., Jones N, Skinner S. (Eds.), Artists Re:Thinking the Blockchain. Torque Editions \& Furtherfield, 287-295, 2017.

[14] N. Jones, S. Skinner, "A Quasi Proto Preface," Catlow R., Garret M., Jones N, Skinner S. (Eds.), Artists Re:Thinking the Blockchain, Torque Editions \& Furtherfield, 9-19, 2017.

[15] A. Alberro, B. Stimson, Conceptual Art: A Critical Anthology, MIT Press, 1999.

[16] M. Zeilinger, "Digital Art as 'Monetized Graphics': enforcing intellectual property on the blockchain," Philos.Technol, 2016, doi: 10.1007/sl3347016-0243-1.

[17] L. Lessig, Free Culture, Penguin, 2004.

[18] A. Moore, K. Himma, "Intellectual Property," Stanford Encyclopedia of Philosophy, http://plato-stanford.edu/entries/intellectual-property/, Retrieved 15 November 2016.

[19] J. Boyle, The Public Domain: Enclosing the Commons of the mind, Yale University Press, 2008.

[20] R. J. Coombe, The Cultural Life of Intellectual Properties: Autorship, Appropriation, and the Law, Duke University Press, 1998.

[21] C. Paul, A Companion to Digital Art, Wiley Blackwell Publishers, 2016.

[22] Y. Hui, "What is a Digital Object?," Metaphilosophy 43(4), 380-395, 2012. doi: 10.1111/j.1467-9973.2012.01761.x.

[23] M. Suvajdzic, D. Stojanovic, J. Appelbaum, "Blockchain Art and Blockchain Facilitated Art Economy: Two Ways in Which Art and Blockchain Collide,"2019 4th Technology Innovation Management and Engineering Science International Conference (TIMES-iCON), 2019, doi: 10.1109/TIMES-iCON47539.2019.9024403. 